Open J. Math. Sci., Vol. 2(2018), No. 1, pp. $18-28$

Website: https://pisrt.org/psr-press/journals/oms/

ISSN: 2523-0212 (Online) 2616-4906 (Print)

http://dx.doi.org/10.30538/oms2018.0014

\title{
M-POLYNOMIALS AND DEGREE-BASED TOPOLOGICAL INDICES OF SOME FAMILIES OF CONVEX POLYTOPES
}

\author{
MUHAMMAD RIAZ, WEI GAO ${ }^{1}$, ABDUL QUDAIR BAIG
}

\begin{abstract}
In this article, we compute closed forms of M-polynomials for three general classes of convex polytopes. From the M-polynomials, we derive degree-based topological indices such as first and second Zagreb indices, modified second Zagreb index, Symmetric division index, etc.

Mathematics Subject Classification: 05C12, 92E10.

Key words and phrases: M-polynomial; degree-based topological index; convex polytope.
\end{abstract}

\section{Introduction}

A graph $G(V, E)$ with vertex set $V(G)$ and edge set $E(G)$ are connected, if there exists a connection between any pair of vertices in $G$. The degree of a vertex is the number of vertices which are connected to that fixed vertex by the edges. In a chemical graph, the degree of any vertex is at most 4 . The distance between two vertices $u$ and $v$ is denoted as $d(u, v)=d_{G}(u, v)$ and is the length of shortest path between $u$ and $v$ in graph $G$. The number of vertices of $G$, adjacent to a given vertex $v$, is the "degree" of this vertex, and will be denoted by $d_{v}$. The concept of degree in graph theory is closely related (but not identical) to the concept of valence in chemistry. For details on basics of graph theory, any standard text such as [1] can be of great help.

Several algebraic polynomials have useful applications in chemistry such as Hosoya polynomial (also called Wiener polynomial) [2], which plays a vital role in determining distance-based topological indices. Among other algebraic polynomials, M-polynomial [3] introduced in 2015, plays the same role in determining

\footnotetext{
Received 06 December 2017. Revised 18 February 2018.

1 Corresponding Author

(C) 2018 Muhammad Riaz, Wei Gao, Abdul Qudair Baig. This is an open access article distributed under the Creative Commons Attribution License, which permits unrestricted use, distribution, and reproduction in any medium, provided the original work is properly cited.
} 
closed form of many degree-based topological indices $[4,5,6,7,8]$. The main advantage of M-polynomial is the wealth of information that it contains about degree-based graph invariants.

Definition 1.1. [3] The M-polynomial of $G$ is defined as:

$$
M(G, x, y)=\sum_{\delta \leq i \leq j \leq \Delta} m_{i j}(G) x^{i} y^{j}
$$

where $\delta=\operatorname{Min}\left\{d_{v} \mid v \in \mathrm{V}(\mathrm{G})\right\}, \Delta=\operatorname{Max}\left\{d_{v} \mid v \in \mathrm{V}(\mathrm{G})\right\}$, and $m_{i j}(G)$ is the edge $v u \in E(G)$ such that $\left\{d_{v}, d_{u}\right\}=\{i, j\}$.

The first topological index was introduced by Wiener [9] and it was named path number, which is now known as Wiener index. In chemical graph theory, this is the most studied molecular topological index due to its wide applications; see for details in [10,11]. Randić index, [12] denoted by $R_{-1 / 2}(G)$ and introduced by Milan Randić in 1975 is also one of the oldest topological index. The Randić index is defined as

$$
R_{-1 / 2}(G)=\sum_{u v \in E(G)} \frac{1}{\sqrt{d_{u} d_{v}}} .
$$

In 1998, working independently, Bollobs and Erds, [13] and Amic et al. [14] proposed the generalized Randić index which has been studied extensively by both chemists and mathematicians [15]. Many mathematical properties have been discussed [16]. For a detailed survey we refer the book [17].

The general Randić index is defined as:

$$
R_{\alpha}(G)=\sum_{u v \in E(G)} \frac{1}{\left(d_{u} d_{v}\right)^{\alpha}},
$$

and the inverse Randić index is defined as $R R_{\alpha}(G)=\sum_{u v \in E(G)}\left(d_{u} d_{v}\right)^{\alpha}$.

Obviously $R_{-1 / 2}(G)$ is the particular case of $R_{\alpha}(G)$ when $\alpha=-\frac{1}{2}$.

The Randic index is the most popular most often applied and most studied among all other topological indices. Many papers and books such as [18, 19, 20] are written on this topological index. Randić himself wrote two reviews on his Randić index [21, 22] and there are three more reviews [23, 24, 25]. The suitability of the Randić index for drug design was immediately recognized, and eventually the index was used for this purpose on countless occasions. The physical reason for the success of such a simple graph invariant is still an enigma, although several more-or-less plausible explanations were offered.

Gutman and Trinajstić introduced first Zagreb index and second Zagreb index, which are defined as: $M_{1}(G)=\sum_{u v \in E(G)}\left(d_{u}+d_{v}\right)$ and $M_{2}(G)=\sum_{u v \in E(G)}\left(d_{u} \times\right.$ $\left.d_{v}\right)$ respectively. The second modified Zagreb index is defined as:

$$
{ }^{m} M_{2}(G)=\sum_{u v \in E(G)} \frac{1}{d(u) d(v)} .
$$

For details about these indices we offer $[26,27,28,29,30]$ for the readers. 
The Symmetric division index is defined as:

$$
\operatorname{SDD}(\mathrm{G})=\sum_{u v \in E(G)}\left\{\frac{\min \left(d_{u}, d_{v}\right)}{\max \left(d_{u}, d_{v}\right)}+\frac{\max \left(d_{u}, d_{v}\right)}{\min \left(d_{u}, d_{v}\right)}\right\} .
$$

Another variant of Randić index is the harmonic index defined as:

$$
H(G)=\sum_{v u \in E(G)} \frac{2}{d_{u}+d_{v}} .
$$

The Inverse sum index is defined as:

$$
I(G)=\sum_{v u \in E(G)} \frac{d_{u} d_{v}}{d_{u}+d_{v}}
$$

The augmented Zagreb index is defined as:

$$
A(G)=\sum_{v u \in E(G)}\left\{\frac{d_{u} d_{v}}{d_{u}+d_{v}-2}\right\}^{3},
$$

and it is useful for computing heat of formation of alkanes [31, 32].

The following Table 1 relates some well-known degree-based topological indices with M-polynoimal [3].

Table 1 Derivation of some degree-based topological indices from

M-polynomial

\begin{tabular}{|l|l|}
\hline Topological Index & Derivation from $M(G ; x, y)$ \\
\hline First Zagreb & $\left(D_{x}+D_{y}\right)(M(G ; x, y))_{x=y=1}$ \\
\hline Second Zagreb & $\left(D_{x} D_{y}\right)(M(G ; x, y))_{x=y=1}$ \\
\hline Second Modified Zagreb & $\left(S_{x} S_{y}\right)(M(G ; x, y))_{x=y=1}$ \\
\hline Randić Index & $\left(D_{x}^{\alpha} D_{y}^{\alpha}\right)(M(G ; x, y))_{x=y=1}$ \\
\hline Inverse Randić Index & $\left(S_{x}^{\alpha} S_{y}^{\alpha}\right)(M(G ; x, y))_{x=y=1}$ \\
\hline Symmetric Division Index & $\left(D_{x} S_{y}+S_{x} D_{y}\right)(M(G ; x, y))_{x=y=1}$ \\
\hline Harmonic Index & $2 S_{x} J(M(G ; x, y))_{x=1}$ \\
\hline Inverse sum Index & $S_{x} J D_{x} D_{y}(M(G ; x, y))_{x=1}$ \\
\hline Augmented Zagreb Index & $S_{x}^{3} Q_{-2} J D_{x}^{3} D_{y}^{3}(M(G ; x, y))_{x=1}$ \\
\hline
\end{tabular}

\section{Main Results}

In this section we give our main results. 
2.1. Computational aspects of Convex Polytopes $T_{n}$. The graph of convex polytope $T_{n}$ can be obtained from the graph of convex polytope $Q_{n}$ by adding new edges. It consists of three-sided faces, five-sided faces and $n$-sided faces. $a_{i+1} b_{i}$, i.e., $V\left(T_{n}\right)=V\left(Q_{n}\right)$ and $V\left(T_{n}\right)=V\left(Q_{n}\right) \cup\left\{a_{i+1} b_{i}: \quad 1 \leq i \leq n\right\}$ as shown in figure 1.

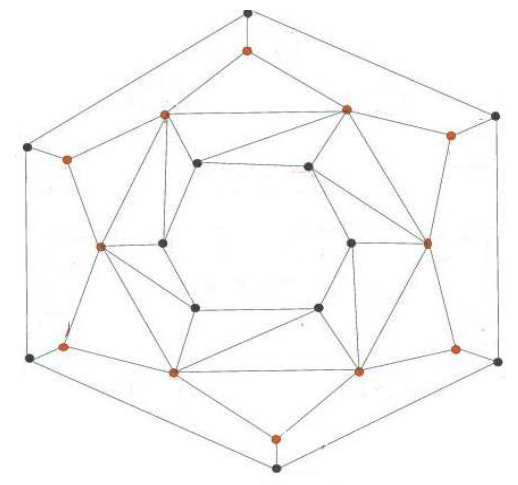

Figure 1. Graph of Convex poltyope $T_{6}$.

Theorem 2.1. Assume we have a convex polytope $T_{n}$, then the M-Polynomial of $T_{n}$ is

$$
M\left(T_{n} ; x, y\right)=2 n x^{3} y^{3}+2 n x^{3} y^{6}+n x^{4} y^{4}+2 n x^{4} y^{6}+n x^{6} y^{6} .
$$

Proof. Let $G=T_{n}$ be a convex polytope. It is easy to see form Figure 1 that

$$
\begin{aligned}
& \left|V\left(T_{n}\right)\right|=4 n, \\
& \left|E\left(T_{n}\right)\right|=8 n .
\end{aligned}
$$

The vertex set of $S_{n}$ has two partitions:

such that

$$
\begin{array}{ll}
V_{1}\left(T_{n}\right)=\left\{u \in V\left(T_{n}\right):\right. & \left.d_{u}=3\right\}, \\
V_{2}\left(T_{n}\right)=\left\{u \in V\left(T_{n}\right):\right. & \left.d_{u}=4\right\}, \\
V_{4}\left(T_{n}\right)=\left\{u \in V\left(T_{n}\right):\right. & \left.d_{u}=6\right\},
\end{array}
$$

$$
\left|V_{1}\left(T_{n}\right)\right|=2 n,\left|V_{2}\left(T_{n}\right)\right|=n,\left|V_{3}\left(T_{n}\right)\right|=n,
$$

The edge set of $T_{n}$ has three partitions:

$$
\begin{gathered}
E_{1}\left(T_{n}\right)=\left\{e=u v \in E\left(T_{n}\right): \quad d_{u}=d_{v}=3\right\}, \\
E_{2}\left(T_{n}\right)=\left\{e=u v \in E\left(T_{n}\right): \quad d_{u}=3, d_{v}=6\right\},
\end{gathered}
$$




$$
\begin{gathered}
E_{3}\left(T_{n}\right)=\left\{e=u v \in E\left(S_{n}\right): \quad d_{u}=d_{v}=4\right\}, \\
E_{4}\left(T_{n}\right)=\left\{e=u v \in E\left(T_{n}\right): \quad d_{u}=4, d_{v}=6\right\}, \\
E_{5}\left(T_{n}\right)=\left\{e=u v \in E\left(T_{n}\right): \quad d_{u}=d_{v}=6\right\},
\end{gathered}
$$

From Figure 1,

$$
\left|E_{1}\left(T_{n}\right)\right|=2 n,\left|E_{2}\left(T_{n}\right)\right|=2 n,\left|E_{3}\left(T_{n}\right)\right|=n,\left|E_{4}\left(T_{n}\right)\right|=2 n,\left|E_{5}\left(T_{n}\right)\right|=n,
$$

From the definition of the M-polynomial

$$
\begin{aligned}
M\left(T_{n} ; x, y\right) & =\sum_{i \leq j} m_{i j}\left(T_{n}\right) x^{i} y^{j} \\
& =\sum_{3 \leq 3} m_{33}\left(T_{n}\right) x^{3} y^{3}+\sum_{3 \leq 6} m_{36}\left(T_{n}\right) x^{3} y^{6}+\sum_{4 \leq 4} m_{44}\left(T_{n}\right) x^{4} y^{4} \\
& +\sum_{4 \leq 6} m_{46}\left(T_{n}\right) x^{4} y^{6}+\sum_{6 \leq 6} m_{66}\left(T_{n}\right) x^{6} y^{6} \\
& =\sum_{u v \in E_{1}} m_{33}\left(T_{n}\right) x^{3} y^{3}+\sum_{u v \in E_{2}} m_{36}\left(T_{n}\right) x^{3} y^{6}+\sum_{u v \in E_{3}} m_{44}\left(T_{n}\right) x^{4} y^{4} \\
& +\sum_{u v \in E_{4}} m_{46}\left(T_{n}\right) x^{4} y^{6}+\sum_{u v \in E_{5}} m_{66}\left(T_{n}\right) x^{6} y^{6} \\
& =\left|E_{1}\right| x^{3} y^{3}+\left|E_{2}\right| x^{3} y^{6}+\left|E_{3}\right| x^{4} y^{4}+\left|E_{4}\right| x^{4} y^{6}+\left|E_{5}\right| x^{6} y^{6} \\
& =2 n x^{3} y^{3}+2 n x^{3} y^{6}+n x^{4} y^{4}+2 n x^{4} y^{6}+n x^{6} y^{6} .
\end{aligned}
$$

Now we compute some degree-based topological indices of double antiprism from this M-polynomial.

Proposition 2.2. Let $T_{n}$ be the double antiprism, then

(1) $M_{1}\left(T_{n}\right)=70 n$.

(2) $M_{2}\left(T_{n}\right)=154 n$

(3) ${ }^{m} M_{2}\left(T_{n}\right)=\frac{73}{144} n$.

(4) $R_{\alpha}\left(T_{n}\right)=2 \times 9^{\alpha} n+2 \times 18^{\alpha} n+16^{\alpha} n+2 \times 24^{\alpha} n+36^{\alpha} n$.

(5) $R R_{\alpha}\left(T_{n}\right)=\frac{2 n}{9 \alpha}+\frac{2 n}{16^{\alpha}}+\frac{n}{16^{\alpha}}+\frac{2 n}{24^{\alpha}}+\frac{n}{36^{\alpha}}$.

(6) $S S D\left(T_{n}\right)=\frac{52}{3} n$.

(7) $H\left(T_{n}\right)=\frac{98}{45} n$.

(8) $I\left(T_{n}\right)=\frac{84}{5} n$.

(9) $A\left(T_{n}\right)==\frac{6534785489}{37044000} n$.

Proof. Let

$$
M\left(T_{n} ; x, y\right)=f(x, y)=2 n x^{3} y^{3}+2 n x^{3} y^{6}+n x^{4} y^{4}+2 n x^{4} y^{6}+n x^{6} y^{6}
$$

Then

$$
D_{x}(f(x, y))=6 n x^{3} y^{3}+6 n x^{3} y^{6}+4 n x^{4} y^{4}+8 n x^{4} y^{6}+6 n x^{6} y^{6},
$$




$$
\begin{gathered}
D_{y}(f(x, y))=6 n x^{3} y^{3}+12 n x^{3} y^{6}+4 n x^{4} y^{4}+12 n x^{4} y^{6}+6 n x^{6} y^{6}, \\
\left(D_{y} D_{x}\right)(f(x, y))=18 n x^{3} y^{3}+36 n x^{3} y^{6}+16 n x^{4} y^{4}+48 n x^{4} y^{6}+36 n x^{6} y^{6}, \\
S_{x} S_{y}(f(x, y))=\frac{2}{9} n x^{3} y^{3}+\frac{1}{9} n x^{3} y^{6}+\frac{1}{16} n x^{4} y^{4}+\frac{1}{12} n x^{4} y^{6}+\frac{1}{36} n x^{6} y^{6}, \\
D_{x}^{\alpha} D_{y}^{\alpha}(f(x, y))=2 \times 9^{\alpha} n x^{3} y^{3}+2 \times 18^{\alpha} n x^{3} y^{6}+16^{\alpha} n x^{4} y^{4}+2 \times 24^{\alpha} n x^{4} y^{6}+36^{\alpha} n x^{6} y^{6}, \\
S_{x}^{\alpha} S_{y}^{\alpha}(f(x, y))=\frac{2 n}{9^{\alpha}} x^{3} y^{3}+\frac{2 n}{16^{\alpha}} x^{3} y^{6}+\frac{n}{16^{\alpha}} x^{4} y^{4}+\frac{2 n}{24^{\alpha}} x^{4} y^{6}+\frac{n}{36^{\alpha}} x^{6} y^{6}, \\
S_{y} D_{x}(f(x, y))=2 n x^{3} y^{3}+n x^{3} y^{6}+n x^{4} y^{4}+\frac{4 n}{3} x^{4} y^{6}+n x^{6} y^{6}, \\
S_{x} D_{y}(f(x, y))=2 n x^{3} y^{3}+4 n x^{3} y^{6}+n x^{4} y^{4}+3 n x^{4} y^{6}+n x^{6} y^{6}, \\
S_{x} J f(x, y)=\frac{n}{3} x^{6}+\frac{n}{4} x^{8}+\frac{2 n}{9} x^{9}+\frac{n}{5} x^{10}+\frac{n}{12} x^{12}, \\
S_{x} J D_{x} D_{y}(f(x, y))==3 n x^{6}+2 n x^{8}+4 n x^{9}+\frac{24}{5} n x^{10}+3 n x^{12}, \\
S_{x}^{3} Q_{-2} J D_{x}^{3} D_{y}^{3} f(x, y)=\frac{1458}{64} n x^{4}+\frac{4096}{216} n x^{6}+\frac{11664}{343} n x^{7}+\frac{27648}{512} n x^{8}+\frac{46656}{1000} n x^{10} .
\end{gathered}
$$

Now from Table 1

(1) $M_{1}\left(T_{n}\right)=\left.\left(D_{x}+D_{y}\right)(f(x, y))\right|_{x=y=1}=70 n$.

(2) $M_{2}\left(T_{n}\right)=\left.D_{x} D_{y}(f(x, y))\right|_{x=y=1}=154 n$.

(3) ${ }^{m} M_{2}\left(T_{n}\right)=\left.S_{x} S_{y}(f(x, y))\right|_{x=y=1}=\frac{73}{144} n$.

(4) $R_{\alpha}\left(T_{n}\right)=\left.D_{x}^{\alpha} D_{y}^{\alpha}(f(x, y))\right|_{x=y=1}=2 \times 9^{\alpha} n+2 \times 18^{\alpha} n+16^{\alpha} n+2 \times$ $24^{\alpha} n+36^{\alpha} n$.

(5) $R R_{\alpha}\left(T_{n}\right)=\left.S_{x}^{\alpha} S_{y}^{\alpha}(f(x, y))\right|_{x=y=1}=\frac{2 n}{9^{\alpha}}+\frac{2 n}{16^{\alpha}}+\frac{n}{16^{\alpha}}+\frac{2 n}{24^{\alpha}}+\frac{n}{36^{\alpha}}$.

(6) $S S D\left(T_{n}\right)=\left.\left(S_{y} D_{x}+S_{x} D_{y}\right)(f(x, y))\right|_{x=y=1}=\frac{52}{3} n$.

(7) $H\left(T_{n}\right)=\left.2 S_{x} J(f(x, y))\right|_{x=1}=\frac{98}{45} n$.

(8) $I\left(T_{n}\right)=S_{x} J D_{x} D_{y}(f(x, y))_{x=1}=\frac{84}{5} n$.

(9) $A\left(T_{n}\right)=\left.S_{x}^{3} Q_{-2} J D_{x}^{3} D_{y}^{3}(f(x, y))\right|_{x=1}=\frac{6534785489}{37044000} n$. 


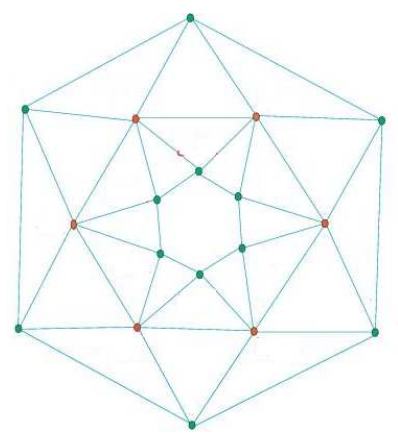

Figure 2. Graph of double antiprism $A_{6}$.

2.2. Computational aspects of Convex Polytopes $A_{n}$. The graph of convex polytope (double antiprism) $A_{n}$ can be obtained from the graph of convex polytope $R_{n}$ Rn by adding new edges $b_{i+1} c_{i}$, i.e.,

$V\left(A_{n}\right)=V\left(R_{n}\right)$ and $V\left(A_{n}\right)=V\left(R_{n}\right) \cup\left\{b_{i+1} c_{i}: \quad 1 \leq \mathrm{i} \leq \mathrm{n}\right\}$ as shown in Figure 2 .

Theorem 2.3. Let $A_{n}$ be the double antiprism, then the M-Polynomial of $A_{n}$ is

$$
M\left(A_{n}, x, y\right)=2 n x^{4} y^{4}+4 n x^{4} y^{6}+n x^{6} y^{6}
$$

Proof. Let $G=A_{n}$ is double antiprism. It is easy to see form figure 2 that

$$
\begin{aligned}
& \left|V\left(A_{n}\right)\right|=3 n, \\
& \left|E\left(A_{n}\right)\right|=7 n .
\end{aligned}
$$

The vertex set of $A_{n}$ has two partitions:

such that

$$
\begin{aligned}
& V_{1}\left(A_{n}\right)=\left\{u \in V\left(A_{n}\right): \quad d_{u}=4\right\}, \\
& V_{2}\left(A_{n}\right)=\left\{u \in V\left(A_{n}\right): \quad d_{u}=6\right\} \text {, }
\end{aligned}
$$

$$
\left|V_{1}\left(A_{n}\right)\right|=2 n,\left|V_{2}\left(A_{n}\right)\right|=n .
$$

The edge set of $A_{n}$ has three partitions:

$$
\begin{gathered}
E_{1}\left(A_{n}\right)=\left\{e=u v \in E\left(A_{n}\right): \quad d_{u}=d_{v}=4\right\}, \\
E_{2}\left(A_{n}\right)=\left\{e=u v \in E\left(A_{n}\right): \quad d_{u}=4, d_{v}=6\right\}, \\
E_{3}\left(A_{n}\right)=\left\{e=u v \in E\left(A_{n}\right): \quad d_{u}=d_{v}=6\right\},
\end{gathered}
$$

From Figure 2,

$$
\left|E_{1}\left(A_{n}\right)\right|=2 n,\left|E_{2}\left(A_{n}\right)\right|=4 n,\left|E_{3}\left(A_{n}\right)\right|=n,
$$


Now from the definition of the M-polynomial

$$
\begin{aligned}
M\left(A_{n}, x, y\right) & =\sum_{i \leq j} m_{i j}\left(A_{n}\right) x^{i} y^{j} \\
& =\sum_{4 \leq 4} m_{44}\left(A_{n}\right) x^{4} y^{4}+\sum_{4 \leq 6} m_{46}\left(A_{n}\right) x^{4} y^{6}+\sum_{5 \leq 5} m_{66}\left(A_{n}\right) x^{6} y^{6} \\
& =\sum_{u v \in E_{1}} m_{44}\left(A_{n}\right) x^{4} y^{4}+\sum_{u v \in E_{2}} m_{46}\left(A_{n}\right) x^{4} y^{6}+\sum_{u v \in E_{3}} m_{66}\left(A_{n}\right) x^{6} y^{6} \\
& =\left|E_{1}\right| x^{4} y^{4}+\left|E_{2}\right| x^{4} y^{6}+\left|E_{3}\right| x^{6} y^{6} \\
& =2 n x^{4} y^{4}+4 n x^{4} y^{6}+n x^{6} y^{6} .
\end{aligned}
$$

Now we compute some degree-based topologcal indices of double antiprism from this M-polynomial.

Proposition 2.4. Let $A_{n}$ be the double antiprism, then

(1) $M_{1}\left(A_{n}\right)=68 \mathrm{n}$.

(2) $M_{2}\left(A_{n}\right)=\frac{23}{72} n$.

(3) ${ }^{m} M_{2}\left(A_{n}\right)=\frac{23}{72} n$.

(4) $R_{\alpha}\left(A_{n}\right)=n\left(4 \times 24^{\alpha}+36^{\alpha}+2 \times 16^{\alpha}\right)$.

(5) $R R_{\alpha}\left(A_{n}\right)=n\left(\frac{2}{16^{\alpha}}+\frac{4}{24^{\alpha}}+\frac{1}{36^{\alpha}}\right)$.

(6) $\operatorname{SSD}\left(A_{n}\right)=\frac{44}{3} n$.

(7) $H\left(A_{n}\right)=\frac{11}{15} n$.

(8) $I\left(A_{n}\right)=\frac{83}{5} n$.

(9) $A\left(A_{n}\right)=\frac{649964}{3375} n$.

2.3. Computational aspects of Convex Polytopes. $S_{n}$ The graph of convex polytope (double antiprism) $S_{n}$ can be obtained from the graph of convex polytope $Q_{n}$ by adding new edges $c_{i} c_{i+1}$, i.e., $V\left(S_{n}\right)=V\left(Q_{n}\right)$ and $V\left(S_{n}\right)=V\left(Q_{n}\right) \cup\left\{c_{i} c_{i+1}: \quad 1 \leq \mathrm{i} \leq \mathrm{n}\right\}$ as shown in Figure 3 .

Theorem 2.5. Let $S_{n}$ be the double antiprism, then the M-Polynomial of $S_{n}$ is

$$
M\left(S_{n} ; x, y\right)=2 n x^{3} y^{3}+2 n x^{3} y^{5}+4 n x^{5} y^{5} .
$$

Proof. Let $G=S_{n}$ be the double antiprism. It is easy to see form Figure 3 that

$$
\begin{aligned}
& \left|V\left(S_{n}\right)\right|=4 n, \\
& \left|E\left(S_{n}\right)\right|=8 n .
\end{aligned}
$$

The vertex set of $S_{n}$ has two partitions:

$$
V_{1}\left(S_{n}\right)=\left\{u \in V\left(S_{n}\right): \quad d_{u}=3\right\},
$$




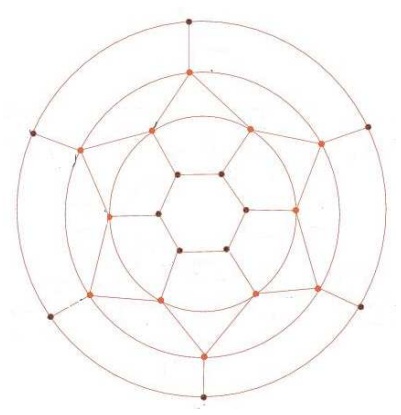

Figure 3. Graph of double antiprism $S_{6}$.

$$
V_{2}\left(S_{n}\right)=\left\{u \in V\left(S_{n}\right): \quad d_{u}=5\right\},
$$

such that

$$
\left|V_{1}\left(S_{n}\right)\right|=2 n,\left|V_{2}\left(S_{n}\right)\right|=2 n .
$$

The edge set of $A_{n}$ has three partitions:

$$
\begin{array}{ll}
E_{1}\left(S_{n}\right)=\left\{e=u v \in E\left(S_{n}\right):\right. & \left.d_{u}=d_{v}=3\right\}, \\
E_{2}\left(S_{n}\right)=\left\{e=u v \in E\left(S_{n}\right):\right. & \left.d_{u}=3, d_{v}=5\right\}, \\
E_{3}\left(S_{n}\right)=\left\{e=u v \in E\left(S_{n}\right):\right. & \left.d_{u}=d_{v}=5\right\}
\end{array}
$$

From Figure 3,

$$
\left|E_{1}\left(S_{n}\right)\right|=2 n,\left|E_{2}\left(S_{n}\right)\right|=2 n,\left|E_{3}\left(S_{n}\right)\right|=4 n,
$$

Now from the definition of the M-polynomial

$$
\begin{aligned}
M\left(S_{n} ; x, y\right) & =\sum_{i \leq j} m_{i j}\left(S_{n}\right) x^{i} y^{j} \\
& =\sum_{u v \in E_{1}} m_{33}\left(S_{n}\right) x^{3} y^{3}+\sum_{u v \in E_{2}} m_{35}\left(S_{n}\right) x^{3} y^{5}+\sum_{u v \in E_{3}} m_{55}\left(S_{n}\right) x^{5} y^{5} \\
& =\left|E_{1}\right| x^{3} y^{3}+\left|E_{2}\right| x^{3} y^{5}+\left|E_{3}\right| x^{5} y^{5} \\
& =2 n x^{3} y^{3}+2 n x^{3} y^{5}+4 n x^{5} y^{5} .
\end{aligned}
$$

Now we compute some degree-based topologcal indices of double antiprism from this M-polynomial.

Proposition 2.6. Let $A_{n}$ be the double antiprism, then 
(1) $M_{1}\left(S_{n}\right)=68 n$.

(2) $M_{2}\left(S_{n}\right)=148 n$.

(3) ${ }^{m} M_{2}\left(S_{n}\right)=\frac{116}{225} n$.

(4) $R_{\alpha}\left(S_{n}\right)=2 n\left(9^{\alpha}+15^{\alpha}+2 \times 25^{\alpha}\right)$.

(5) $R R_{\alpha}\left(S_{n}\right)=2 n\left(\frac{1}{9^{\alpha}}+\frac{1}{15^{\alpha}}+\frac{2}{25^{\alpha}}\right)$.

(6) $S S D\left(S_{n}\right)=\frac{248}{15} n$.

(7) $H\left(S_{n}\right)=\frac{59}{60} n$.

(8) $I\left(S_{n}\right)=\frac{67}{4} n$.

(9) $A\left(S_{n}\right)=\frac{22541}{128} n$.

\section{Conclusions and Discussions}

We computed closed forms of M-polynomial of three general classes of convex polytopes at first. Then we derived as many as nine degree-based topological indices such as first and second Zagreb indices, modified second Zagreb index, Symmetric division index, Augmented Zagreb index, Inverse-sum index etc.

\section{Competing Interest}

The authors declare no competing interest.

\section{REFERENCES}

1. Brckler, F. M., Dolic, T., Graovac, A., \& Gutman, I. (2011). On a class of distance-based molecular structure descriptors. Chemical physics letters, 503(4-6), 336-338.

2. Gutman, I. (1993). Some properties of the Wiener polynomial. Graph Theory Notes New York, 25, 13-18.

3. Deutsch, E., \& Klavar, S. (2015). M-polynomial and degree-based topological indices. Iranian Journal of Mathematical Chemistry, 6(2), 93-102.

4. Munir, M., Nazeer, W., Rafique, S., \& Kang, S. M. (2016). M-polynomial and related topological indices of Nanostar dendrimers. Symmetry, 8(9), 97.

5. Munir, M., Nazeer, W., Rafique, S., \& Kang, S. M. (2016). M-polynomial and related topological indices of Nanostar dendrimers. Symmetry, 8(9), 97.

6. Munir, M., Nazeer, W., Rafique, S., \& Kang, S. M. (2016). M-polynomial and degree-based topological indices of polyhex nanotubes. Symmetry, 8(12), 149.

7. Munir, M., Nazeer, W., Rafique, S., Nizami, A. R., \& Kang, S. M. (2016). Some computational aspects of triangular Boron nanotubes. doi:10.20944/preprints201611.0041.v1.

8. Munir, M., Nazeer, W., Shahzadi, Z., \& Kang, S. M. (2016). Some invariants of circulant graphs. Symmetry, 8(11), 134.

9. Wiener, H. (1947). Structural determination of paraffin boiling points. Journal of the American Chemical Society, 69(1), 17-20.

10. Dobrynin, A. A., Entringer, R., \& Gutman, I. (2001). Wiener index of trees: theory and applications. Acta Applicandae Mathematica, 66(3), 211-249.

11. Gutman, I. \& Polansky, O. E. (1986). Mathematical Concepts in Organic Chemistry. Springer-Verlag New York, USA.

12. Randic, M. (1975). Characterization of molecular branching. Journal of the American Chemical Society, 97(23), 6609-6615.

13. Bollobs, B., \& Erds, P. (1998). Graphs of extremal weights. Ars Combinatoria, 50, 225-233.

14. Amić, D., Belo, D., Lucić, B., Nikolic, S., \& Trinajstic, N. (1998). The vertex-connectivity index revisited. Journal of chemical information and computer sciences, 38(5), 819-822. 
15. Hu, Y., Li, X., Shi, Y., Xu, T., \& Gutman, I. (2005). On molecular graphs with smallest and greatest zeroth-order general Randic index. MATCH Commun. Math. Comput. Chem, $54(2), 425-434$.

16. Caporossi, G., Gutman, I., Hansen, P., \& Pavlovic, L. (2003). Graphs with maximum connectivity index. Computational Biology and Chemistry, 27(1), 85-90.

17. Li, X., \& Gutman, I. (2006). Mathematical Chemistry Monographs No. 1. University of Kragujevac, Kragujevac, Macedonia.

18. Kier, L. B., \& Hall, L. H. (1976). Molecular Connectivity in Chemistry and Drug Research Academic. New York.

19. Kier, L. B., \& Hall, L. H. (1986). Molecular connectivity in structure-activity analysis. Wiley, New York.

20. Li, X., \& Gutman, I. (2006). Mathematical aspects of Randic-type molecular structure descriptors, Math. Chem. Monographs, (1).

21. Gutman, I. \& Furtula, B. (2008). Recent Results in the Theory of Randić Index. University of Kragujevac, Kragujevac, Macedonia.

22. Randic, M. (2008). On history of the Randic index and emerging hostility toward chemical graph theory. MATCH Commun. Math. Comput. Chem, 59, 5-124.

23. Randić, M. (2001). The connectivity index 25 years after. Journal of Molecular Graphics and Modelling, 20(1), 19-35.

24. Li, X., \& Shi, Y. (2008). A survey on the Randic index. MATCH Commun. Math. Comput. Chem, 59(1), 127-156.

25. Li, X., Shi, Y., \& Wang, L. (2008). An updated survey on the Randic index. Recent Results in the Theory of Randic Index, University of Kragujevac and Faculty of Science Kragujevac, 9-47.

26. Nikolic, S., Kovacevic, G., Milicevic, A., \& Trinajstic, N. (2003). The Zagreb indices 30 years after. Croatica chemica acta, 76(2), 113-124..

27. Gutman, I., \& Das, K. C. (2004). The first Zagreb index 30 years after. MATCH Commun. Math. Comput. Chem, 50, 83-92.

28. Das, K. C., \& Gutman, I. (2004). Some properties of the second Zagreb index. MATCH Commun. Math. Comput. Chem, 52(1), 3-1.

29. Zhou, B. (2004). Zagreb indices. MATCH-Communications in Mathematical and in Computer Chemistry, (52), 113-118.

30. Vukicevic, D., \& Graovac, A. (2004). Valence connectivity versus Randic, Zagreb and modified Zagreb index: A linear algorithm to check discriminative properties of indices in acyclic molecular graphs. Croatica chemica acta, 77(3), 501-508.

31. Huang, Y., Liu, B., \& Gan, L. (2012). Augmented Zagreb index of connected graphs. Match-Communications in Mathematical and Computer Chemistry, 67(2), 483.

32. Furtula, B., Graovac, A. \& Vukicević, D. (2010). Augmented Zagreb index. J. Math. Chem. $48,370-380$.

\section{Muhammad Riaz}

Department of Mathematics and Statistics, The University of Lahore, Pakistan.

e-mail: mriazshah@gmail.com

Wei Gao

School of Information Science and Technology,Yunnan Normal University, Kunming 650500, China.

e-mail: gaowei@ynnu.edu.cn

\section{Abdul Qudair Baig}

Department of Mathematics, The University of Lahore, Pakpattan Campus, Pakistan. e-mail:aqbaig1@gmail.com 